\title{
A NOTE ON COMONOTONIC ADDITIVITY
}

\author{
by JUNE M. PARKER
}

(Received 6 December, 1994)

\begin{abstract}
The axiom of comonotonic independence for a preference ordering was introduced by Schmeidler [9]. It leads to the comonotonic additivity for the functional representing the preference ordering, which is necessarily a Choquet integral.

The aim of this paper is to illuminate the concepts of comonotonicity, comonotonic independence and comonotonic additivity. For example the seemingly weaker condition of weak comonotonic independence used by Chateauneuf in [2] is seen to be equivalent to comonotonic independence. Comonotonic additivity is characterized as additivity on chains of sets. From this the characterization of Choquet integrals in [4], [1], [8] follows easily.
\end{abstract}

1. Introduction. This paper was motivated by an interest in the representation of preference orderings by generalizations of expected utility. Several recent papers ([8], [2]) have included axioms of comonotonic independence for these preference orderings. The aim here is to make transparent the comonotonicity relationship and the nature of comonotonic independence.

The framework is the simple one chosen by Chateauneuf in [2] where acts are just real-valued functions on a set of states of nature, $\Omega$. Two such functions on $\Omega$ are comonotonic if they are mutually reinforcing, in the sense that a large value of one of them at a given point of $\Omega$ is not cancelled out by small value of the other (see Lemma 1 below). As Schmeidler points out in [9], the decision that an act $f$ is preferred to an act $g$ depends upon information about subsets of $\Omega$. Roughly speaking, if on a subset of $\Omega$, which is known to occur with high probability, the values of $f$ are more favourable than the values of $g$, then it is likely that $f$ will be preferred to $g$.

If "more favourable" means "larger", it seems natural, for an act $f$, to look at sets of the form $\{f \geq \alpha\}=\{\omega \in \Omega: f(\omega) \geq \alpha\}$, for $\alpha \in \mathbb{R}$. This reveals the essential nature of the comonotonicity relationship. Two functions $f, g: \Omega \rightarrow \mathbb{R}$ are comonotonic if and only if for all $\alpha, \beta \in \mathbb{R}$ the subsets $\{f \geq \alpha\}$ and $\{g \geq \beta\}$ of $\Omega$ form a chain (see Proposition 4). Denneberg [5] uses this property to define comonotonicity.

Suppose, now, that there is a preference ordering $\leq$ on the set of acts and that $\leq$ may be represented by a functional $l$ in the sense that $f \leq g$ if and only if $I(f) \leq I(g)$. The axiom of comonotonic independence would require that $I(f+g)=I(f)+I(g)$ for comonotonic acts $f$ and $g$, that is, that $I$ be additive on comonotonic functions. This seems to be a reasonable requirement, given that comonotonic functions can be thought of as being mutually reinforcing in the sense of Lemma 1.

The main result is Theorem 7 , which shows that, for a functional $I$, the property of additivity on comonotonic functions is equivalent to additivity on chains of subsets of $\Omega$. The importance of behaviour on chains of sets for Choquet integrals was brought out in [1] and [10]. The characterization of Choquet integrals ([4], [1] and [8]), in terms of additivity on comonotonic functions, follows easily from Theorem 7 .

Glasgow Math. J. 38 (1996) 199-205. 
2. Comonotonic functions. Let $\Omega$ be a non-empty set and $f, g: \Omega \rightarrow \mathbb{R}$. The functions $f$ and $g$ are said to be comonotonic (see [8]) if for all $s, t \in \Omega$

$$
(f(s)-f(t))(g(s)-g(t)) \geq 0 .
$$

The definition can be reformulated as follows.

LEMMA 1. Functions $f$ and $g: \Omega \rightarrow \mathbb{R}$ are comonotonic if and only if for all $s, t \in \Omega$

$$
f(s)>f(t) \text { implies } g(s) \geq g(t) .
$$

Some properties of comonotonicity are immediately clear: any function $f$ and any constant function are comonotonic. Also if $f$ and $g$ are comonotonic then so are $\alpha f$ and $g$ for all $\alpha>0$. For the case that $\Omega=\mathbb{R}$, if the functions $f, g: \mathbb{R} \rightarrow \mathbb{R}$ are both monotonic non-decreasing (or both non-increasing) then they are comonotonic. The next lemma gives some indication of the ways in which comonotonic functions may be combined.

Lemma 2. For functions $f, g, h: \Omega \rightarrow \mathbb{R}$;

(a) if $f$ and $h$ are comonotonic and $g$ and $h$ are comonotonic, then $f+g$ and $h$ are comonotonic;

(b) if $f, g$ and $h$ are pairwise comonotonic, then $\max \{f, g\}$ and $h$ are comonotonic, as are $\min \{f, g\}$ and $h$.

Comonotonicity is not transitive, as can easily be seen by considering indicator functions of subsets $\Omega$. If $C \subseteq \Omega$, its indicator function $1_{C}: \Omega \rightarrow \mathbb{R}$ is defined by: $1_{C}(s)=1$ if $s \in C$ and $1_{C}(s)=0$ if $s \in \Omega \backslash C$. $B \subseteq A$.

Lemma 3. Let $A, B \subseteq \Omega$.Then $1_{A}$ and $1_{B}$ are comonotonic if and only if $A \subseteq B$ or

A family $\mathscr{C}$ of subsets of $\Omega$ is called a chain if for all $C_{1}, C_{2} \in \mathscr{C}$ either $C_{1} \subseteq C_{1}$ or $C_{2} \subseteq C_{1}$. For a finite family $\mathscr{C}$ with $n$ elements this means that the members can be labelled $C_{1}, C_{2}, \ldots, C_{n}$ in such a way that $C_{1} \supseteq C_{2} \supseteq \ldots \supseteq C_{n}$.

The lemma above generalizes to give the next result, which pinpoints the nature of the comonotonicity relationship. The following, together with further characterizations of comonotonicity can be found in [5, Proposition 4.5$]$.

Proposition 4. The functions $f$ and $g: \Omega \rightarrow \mathbb{R}$ are comonotonic if and only if, for all $\alpha, \beta \in \mathbb{R}$, the subsets $\{f \geq \alpha\}$ and $\{g \geq \beta\}$ of $\Omega$ form a chain.

REMARK. This is equivalent to the condition that the family of all subsets of $\Omega$ taking one of the four forms $\{f \geq \alpha\},\{f>\alpha\},\{g \geq \beta\}$ or $\{g>\beta\}$ for some $\alpha$ or $\beta \in \mathbb{R}$ be a chain.

Proof. Suppose that, for all $\alpha, \beta \in \mathbb{R}$, the sets $\{f \geq \alpha\}$ and $\{g \geq \beta\}$ form a chain. Let $s, t \in \Omega$ be such that $f(s)>f(t)$ and set $\alpha=f(s)$ and $\beta=g(t)$. Since $t \notin\{f \geq \alpha\}$ and $t \in\{g \geq \beta\}$ it must be that $\{f \geq \alpha\} \subseteq\{g \geq \beta\}$. It follows that $s \in\{g \geq \beta\}$, that is $g(s) \geq g(t)$. By Lemma $1, f$ and $g$ are comonotonic. 
Now suppose that there exist $\alpha, \beta \in \mathbb{R}$ such that $\{f \geq \alpha\}$ and $\{g \geq \beta\}$ do not form a chain. Then there exist $s, t \in \Omega$ such that $s \in\{f \geq \alpha\} \backslash\{g \geq \beta\}$ and $t \in\{g \geq \beta\} \backslash\{f \geq \alpha\}$. This means that $f(s) \geq \alpha>f(t)$ and $g(t) \geq \beta>g(s)$, so that $f$ and $g$ are not comonotonic.

3. Integral-like functionals. Consider $B(\Omega)$ the space of all bounded functions $f: \Omega \rightarrow \mathbb{R}$ with the supremum norm: for $f \in B(\Omega)$ the norm of $f$ is $\|f\|=\sup _{s \in \Omega}|f(s)|$.

Let $\mathscr{A}$ be a $\sigma$-algebra of subsets of $\Omega$. A finite linear combination of indicator functions $f=\sum_{i=1}^{n} \alpha_{i} 1_{A_{i}}$ with $\alpha_{1}, \alpha_{2}, \ldots, \alpha_{n} \in \mathbb{R}$ and $A_{1}, A_{2}, \ldots, A_{n} \in \mathscr{A}$ is called a simple function. It is worth noting that every simple function $f=\sum_{i=1}^{n} \alpha_{i} 1_{A_{i}}$, with $\alpha_{1}, \alpha_{2}, \ldots, \alpha_{n} \in$ $\mathbb{R}$ and $A_{1}, A_{2}, \ldots, A_{n} \in \mathscr{A}$, can be written in "chain" form: $f=\sum_{j=1}^{m} \gamma_{j} 1_{c_{j}}$ with $\gamma_{1}, \gamma_{2}, \ldots, \gamma_{m} \in \mathbb{R}$ and $C_{1} \supseteq C_{2} \supseteq \cdots \supseteq C_{m}$ in $\mathscr{A}$ (see [10]). For this function, the sets $\{f \geq \alpha\}$ for $\alpha \in \mathbb{R}$ are just $\Omega, C_{1}, C_{2}, \ldots, C_{m}, \varnothing$.

A function $f: \Omega \rightarrow \mathbb{R}$ is said to be measurable if $f^{-1}(J) \in \mathscr{A}$ for all sub-intervals $J$ of $\mathbb{R}$. Clearly every simple function is measurable.

The reason for choosing $\mathscr{A}$ to be a $\sigma$-algebra rather than just an algebra is that the family of bounded measurable functions then coincides with the Banach space $B(\Omega, \mathscr{A})$ which is the closure in $B(\Omega)$, with respect to the supremum norm, of the family of all simple functions. It is sometimes overlooked, that $f+g$ need not be measurable for all measurable $f, g: \Omega \rightarrow \mathbb{R}$, if $\mathscr{A}$ is an algebra but not a $\sigma$-algebra. An alternative way round this difficulty would be to use the weaker definition of measurability, with respect to a non-empty collection of subsets of $\Omega$, which is given in [7]. The resulting family of measurable functions is closed under addition of comonotonic functions.

The next lemma shows that if $f$ and $g$ are comonotonic then $f$ may be approximated by simple functions, each of which is comonotonic with $g$.

Lemma 5. Let $f, g$ be comonotonic functions in $B^{+}(\Omega, \mathscr{A})$. Then there exists a sequence $\left(f_{n}\right)_{n \in \mathbb{N}}$ of simple functions in $B^{+}(\Omega, \mathscr{A})$, which is norm convergent to $f$, such that, for each $n \in \mathbb{N}$, the functions $f_{n}$ and $g$ are comonotonic and $\left\{f_{n}>0\right\}=\{f>0\}$.

Proof. For $n \in \mathbb{N}$ define

$$
f_{n}=\sum_{k=1}^{2^{n}} \frac{\|f\|}{2^{n}} 1_{C_{k}} \quad \text { where } \quad C_{k}=\left\{f>\frac{(k-1)\|f\|}{2^{n}}\right\}, \quad k=1,2, \ldots, 2^{n} .
$$

Then $\left(f_{n}\right)_{n \in \mathbb{N}}$ is norm-convergent to $f$, and for each $n \in \mathbb{N}\left\{f_{n}>0\right\}=\{f>0\}$. Further $f_{n}$ and $g$ are comonotonic, for each $n \in \mathbb{N}$, by the characterization in Theorem 4 .

Definition 1. Let $B^{+}(\Omega, \mathscr{A})$ be the cone of all functions in $B(\Omega, \mathscr{A})$ which assume only non-negative values. An integral-like functional on $B^{+}(\Omega, \mathscr{A})$ is a mapping $I: B^{+}(\Omega, \mathscr{A}) \rightarrow \mathbb{R}$ such that

(i) $I$ is monotone: if $f, g \in B^{+}(\Omega, \mathscr{A})$ and $f \leq g$ then $I(f) \leq I(g)$,

(ii) $I$ is positive homogeneous: if $f \in B^{+}(\Omega, \mathscr{A})$ and $\gamma \geq 0$ then $I(\gamma f)=\gamma I(f)$.

$I$ is said to be additive on comonotonic functions if $I(f+g)=I(f)+I(g)$ for all comonontoic $f, g \in B^{+}(\Omega, \mathscr{A})$. $I$ is said to be $C$-additive if $I\left(\gamma 1_{\Omega}+f\right)=\gamma I\left(1_{\Omega}\right)+I(f)$ for all $f \in B^{+}(\Omega, \mathscr{A})$ and all $\gamma \geq 0$. 
The properties of additivity on comonotonic functions and of $C$-additivity correspond to those of comonotonic independence and certainty independence used in the papers [8], [2] and [6] respectively.

Since a constant function and any other function are comonotonic, $C$-additivity is implied by comonotonic additivity. The next lemma is well-known: for example, see [6, Lemma 3.4].

Lemma 6. If I is a C-additive integral-like functional then I is uniformly continuous with respect to the norm topology on $B^{+}(\Omega, \mathscr{A})$.

Proof. Let $f, g \in B^{+}(\Omega, \mathscr{A})$. Then

$$
\begin{aligned}
I(f)-I(g) & \leq I\left(\|f-g\| 1_{\Omega}+g\right)-I(g) \\
& =\|f-g\| I\left(1_{\Omega}\right)+I(g)-I(g) \\
& =\|f-g\| I\left(1_{\Omega}\right) .
\end{aligned}
$$

Similarly $I(g)-I(f) \leq\|f-g\| I\left(1_{\Omega}\right)$ so that $|I(f)-I(g)| \leq\|f-g\| I\left(1_{\Omega}\right)$.

Theorem 7. Let $I$ be an integral-like functional on $B^{+}(\Omega, \mathscr{A})$. Then the following conditions are equivalent:

(i) $I$ is additive on comonotonic functions;

(ii) for all $f \in B^{+}(\Omega, \mathscr{A}), \gamma \geq 0$ and all $C \in \mathscr{A}$ such that $\{f>0\} \subseteq C$,

$$
I\left(\gamma 1_{C}+f\right)=\gamma I\left(1_{C}\right)+I(f) ;
$$

(iii) I is uniformly continuous with respect to the norm-topology on $B^{+}(\Omega, \mathscr{A})$ and, for all finite chains $C_{1} \supseteq C_{2} \supseteq \cdots \supseteq C_{m}$ in $\mathscr{A}$, and all $\gamma_{1}, \gamma_{2}, \ldots, \gamma_{m} \geq 0$,

$$
I\left(\sum_{j=1}^{m} \gamma_{j} 1_{C_{j}}\right)=\sum_{j=1}^{m} \gamma_{j} I\left(1_{C_{j}}\right)
$$

(iv) $I$ is uniformly continuous with respect to the norm-topology on $B^{+}(\Omega, \mathscr{A})$ and, for all finite chains $C_{1} \supseteq C_{2} \supseteq \cdots \supseteq C_{m}$ in $\mathscr{A}$,

$$
I\left(\sum_{j=1}^{m} 1_{C_{j}}\right)=\sum_{j=1}^{m} I\left(1_{C_{j}}\right)
$$

Proof. (ii) follows from (i) because $f$ and $1_{C}$ are comonotonic.

If (ii) is assumed then $I$ is uniformly continuous with respect to the norm by the previous lemma, since $I$ is, in particular, $C$-additive. The equation in (iii) follows by induction on $m$ : for $m=1$ it holds because $I$ is an integral-like functional. Suppose it holds for $m=k$ and consider $\sum_{j=1}^{k+1} \gamma_{j} 1_{C_{j}}$ with $C_{1} \supseteq C_{2} \supseteq \cdots \supseteq C_{k+1}$ in $\mathscr{A}$, and $\gamma_{1}, \gamma_{2}, \ldots, \gamma_{k+1} \geq 0$. Choose $f=\sum_{i=2}^{k+1} \gamma_{j} 1_{C_{j}}$; then, by inductive assumption, $I(f)=\sum_{j=2}^{k+1} \gamma_{j} I\left(1_{C_{j}}\right)$ and by (ii)

$$
I\left(\sum_{j=1}^{k+1} \gamma_{j} 1_{C_{i}}\right)=I\left(\gamma_{1} 1_{C_{1}}+f\right)=\gamma_{1} I\left(1_{C_{1}}\right)+I(f)=\sum_{j=1}^{k+1} \gamma_{j} I\left(1_{C_{j}}\right)
$$

This proves the equation in (iii) for $m=k+1$.

To prove that (i) follows from (iii), notice first, for $f, g$ comonotonic simple functions 
in $B^{+}(\Omega, \mathscr{A})$, that (iii) already implies $I(f+g)=I(f)+I(g)$. For, suppose that $f=$ $\sum_{j=1}^{m} \gamma_{j} 1_{C}$, and $g=\sum_{k=1}^{n} \delta_{k} 1_{D_{k}}$ are the two functions in "chain" form; then the comonotonicity of $f$ and $g$ means that the collection $\left\{C_{1}, C_{2}, \ldots, C_{m}, D_{1}, D_{2}, \ldots, D_{n}\right\}$ is a chain. Hence, by (iii)

$$
I(f+g)=\sum_{j=1}^{m} \gamma_{j} I\left(1_{C_{j}}\right)+\sum_{k=1}^{n} \delta_{k} I\left(1_{D_{k}}\right)=I(f)+I(g) .
$$

Now let $g$ be a simple function and approximate $f \in B(\Omega, \mathscr{A})$ by a sequence $\left(f_{n}\right)_{n \in \mathbb{N}}$ with the properties described in Lemma 5. Then $I\left(f_{n}+g\right)=I\left(f_{n}\right)+I(g)$ for all $n \in \mathbb{N}$ so that $I(f+g)=I(f)+I(g)$ by the continuity of $I$. This procedure can be repeated for $f, g \in B(\Omega, \mathscr{A})$, approximating $g$ according to Lemma 5. This completes the proof that (iii) implies (i).

It remains to show that (iii) and (iv) are equivalent. Clearly (iv) follows from (iii). Assume (iv); this already implies the equation in (iii) for the case that $\gamma_{1}, \gamma_{2}, \ldots, \gamma_{n}$ are non-negative integers. Now suppose that $\gamma_{1}, \gamma_{2}, \ldots, \gamma_{n} \geq 0$ are rational and let $k \in \mathbb{N}$ be such that $k \gamma_{1}, k \gamma_{2}, \ldots, k \gamma_{n}$ are integers. Then

$$
I\left(\sum_{j=1}^{m} \gamma_{j} 1_{C_{i}}\right)=\frac{1}{k} I\left(\sum_{j=1}^{m} k \gamma_{j} 1_{C_{j}}\right)=\frac{1}{k} \sum_{j=1}^{m} k \gamma_{j} I\left(1_{C_{j}}\right)=\sum_{j=1}^{m} \gamma_{j} I\left(1_{C_{j}}\right) .
$$

The equation now follows for any real non-negative $\gamma_{1}, \gamma_{2}, \ldots, \gamma_{n}$, since the rationals are dense in $\mathbb{R}$ and by the continuity of $I$.

The condition (ii) in the theorem above is the analogue for functionals of the axiom of weak comonotonic independence in [2]. Another condition which is equivalent to those in Theorem 7 is the weak additivity in [1, equations (9) and (10)]. The equivalence of (i) and (iii), as will be seen in the next section, is the essence of the characterization ([4], [1] and $[\mathbf{8}]$ ) of the Choquet integral in terms of additivity on comonotonic functions.

4. The Choquet integral. Let $v: \mathscr{A} \rightarrow[0, \infty)$ be a set function with $v(\varnothing)=0$ which is monotone; that is, for $A, B \in \mathscr{A}, A \subseteq B$ implies $v(A) \leq v(B)$. The Choquet integral, introduced in [3], associated with $v$ is the functional $I_{v}$ on $B^{+}(\Omega, \mathscr{A})$ defined by

$$
I_{v}(f)=\int_{0}^{\infty} v(\{f \geq x\}) d x \text { for all } f \in B^{+}(\Omega, \mathscr{A}) .
$$

Notice that $I_{v}$ is an integral-like functional.

The second property of $l_{v}$ listed in the next lemma can be found in [10] and [1]; the first is well known.

LEMMA 8. Let $I_{v}$ be the Choquet integral associated with the monotone set function $v$.

(a) $I_{v}$ is $C$-additive, hence uniformly continuous with respect to the norm-topology on $B^{+}(\Omega, \mathscr{A})$.

(b) For $f=\sum_{i=1}^{n} \gamma_{i} 1_{C_{i}}$ with $\gamma_{1}, \gamma_{2}, \ldots, \gamma_{n} \geq 0$ and $C_{1} \supseteq C_{2} \supseteq \cdots \supseteq C_{n}$ in $\mathscr{A}$,

$$
I_{v}(f)=\sum_{i=1}^{n} \gamma_{i} v\left(C_{i}\right)
$$


Proof. (a) Let $f \in B(\Omega, \mathscr{A})$ and $\gamma \geq 0$; then for $x \in[0, \infty)$

$$
\left\{f+\gamma 1_{\Omega} \geq x\right\}= \begin{cases}\Omega & \text { if } 0 \leq x<\gamma \\ \{f \geq x-\gamma\} & \text { if } x \geq \gamma .\end{cases}
$$

Hence $I_{v}\left(f+\gamma 1_{\Omega}\right)=\int_{\gamma}^{\gamma} v(\Omega) d x+\int_{\gamma}^{\infty} v(\{f \geq x-\gamma\}) d x=\gamma I_{v}\left(1_{\Omega}\right)+I_{v}(f)$.

(b) Define the intervals $J_{1}=\left(0, \gamma_{1}\right]$ and $J_{k}=\left(\sum_{i=1}^{k-1} \gamma_{i}, \sum_{i=1}^{k} \gamma_{i}\right]$, for $k=2,3, \ldots, n$. Then for $x \in[0, \infty)$

Hence

$$
\{f \geq x\}= \begin{cases}\Omega & \text { if } x=0 \\ C_{k} & \text { if } x \in J_{k} \text { for } k=1,2, \ldots, n \\ \varnothing & \text { if } x>\sum_{i=1}^{n} \gamma_{i} .\end{cases}
$$

$$
I_{v}(f)=\sum_{k=1}^{n} \int_{J_{k}} v\left(C_{k}\right) d x=\sum_{k=1}^{n} \gamma_{k} v\left(C_{k}\right)
$$

The following result may now be proved using Theorem 7 .

Theorem 9 [4, page 81], [1, Theorem 3], [8, Proposition 2]. Let I be an integral-like functional on $B^{+}(\Omega, \mathscr{A})$, and let $v$ be the set function on $\mathscr{A}$ defined by $v(A)=I\left(1_{A}\right)$ for all $A \in \mathscr{A}$. Then $I$ is the Choquet integral associated with the set function $v$ if and only if $I$ is additive on comonotonic functions.

Proof. That $I_{v}$ is additive on comonotonic functions is a direct consequence of Theorem 7 and the above lemma. By Theorem 7 if $I$ is additive on comonotonic functions then $I$ is uniformly norm-continuous and its values are fixed on simple functions by (iii) of that theorem. By the lemma above these values coincide with the values of $I_{v}$ on the simple functions. Since $I_{v}$ is also uniformly norm-continuous and the simple functions are dense in $B^{+}(\Omega, \mathscr{A})$, the two functionals $I$ and $I_{v}$ are equal.

\section{REFERENCES}

1. B. Anger, Representation of capacities, Math. Ann. 229 (1977), 245-258.

2. A. Chateauneuf, On the use of capacities in modelling uncertainty aversion and risk aversion, J. Math. Economics 20 (1991), 343-369.

3. G. Choquet, Theory of capacities, Ann. Inst. Fourier 5 (1953-4), 131-295.

4. C. Dellacherie, Quelques commentaires sur les prolongements de capacités, Séminaire de Probabilités V, Strasbourg, Lecture Notes in Mathematics 191 (Springer-Verlag, 1970), 77-81.

5. D. Denneberg, Non-additive Measure and Integral (Kluwer, 1994).

6. I. Gilboa, D. Schmeidler, Maxmin expected utility with non-unique prior, J. Math. Economics 18 (1989), 141-153.

7. G. Greco, Sur la mesurabilité d'une fonction numerique par rapport à une famille d'ensembles, Rend. Sem. Mat. Univ. Padova 65 (1981), 163-176.

8. D. Schmeidler, Integral representation without additivity, Proc. Amer. Math. Soc. 97 (1986), 255-261. 
9. D. Schmeidler, Subjective probability and expected utility without additivity, Econometrica 57 (1989), 571-587.

10. F. Topsøe, On construction of measures, Proc. conference, Topology and Measure I, Zinnowitz, Ernst-Moritz-Arndt Univ., Greifswald, 1978, 343-381.

School of Mathematics

UNIVERSITY OF HULL

COTTINGHAM ROAD

HULL HU6 7RX. 